\title{
Brain Dysfunction in Patients with Chronic Critical Illness
}

\author{
Timothy D Girard MD MSCI
}

\author{
Introduction \\ Coma and Delirium During Critical Illness \\ Long-Term Brain Dysfunction in Survivors of Critical Illness \\ Management of Brain Dysfunction During Critical Illness \\ Future Directions \\ Summary
}

\begin{abstract}
Critically ill patients frequently experience acute brain dysfunction in the form of coma or delirium, both of which are common during acute and chronic critical illness (CCI). These manifestations of brain dysfunction are associated with numerous adverse outcomes during acute critical illness, including prolonged hospitalization, increased healthcare costs, and increased mortality. The prognosis of CCI patients with coma or delirium has not yet been thoroughly studied, but preliminary studies suggest this population is at high risk for detrimental outcomes associated with acute brain dysfunction. Additionally, a high percentage of patients who survive acute or CCI suffer from long-term brain dysfunction, which manifests primarily as memory deficits and executive dysfunction and is predicted by brain dysfunction in the ICU. Interventions directed at reducing the burden of brain dysfunction during critical illness have shown promise in studies of patients with acute critical illness, but these therapies have yet to be studied during CCI. Thus, multicenter randomized trials are needed to determine which interventions are most effective for such patients. Until these data are available, management strategies that have been proven beneficial during acute critical illness-such as reduction of sedative exposure, especially to benzodiazepines, and early use of physical and occupational therapy-should be employed during the treatment of patients with CCI. Key words: brain dysfunction; coma; delirium; critical illness; chronic critical illness. [Respir Care 2012;57(6):947-955. (c) 2012 Daedalus Enterprises]
\end{abstract}

\section{Introduction}

Brain dysfunction is one of the most common forms of organ failure complicating critical illness. Acutely, brain dysfunction during critical illness most often manifests as coma or delirium, with coma being a state of unarousable

\footnotetext{
Timothy D Girard MD MSCI is affiliated with the Division of Allergy, Pulmonary, and Critical Care Medicine, and the Center for Health Services Research, Department of Medicine, Vanderbilt University School of Medicine, Nashville, Tennessee, and with the Geriatric Research, Education, and Clinical Center Service, Department of Veterans Affairs Medical Center, Tennessee Valley Healthcare System, Nashville, Tennessee.

Dr Girard presented a version of this paper at the 49th RESPIRATORY CARE Journal Conference, "The Chronically Critically Ill Patient," held September 9-10, 2011, in St Petersburg, Florida.
}

unresponsiveness, ${ }^{1}$ and delirium being an acute and fluctuating disturbance of both consciousness and cognition. ${ }^{2}$ Both forms of brain dysfunction can result from a variety of neurologic insults, including ischemic or traumatic brain injury, toxic or metabolic derangements, or as side effects of medications, especially some sedative agents frequently used in the ICU.

\footnotetext{
Dr Girard was partly supported by grant AG034257 from the National Institutes of Health. Dr Girard has disclosed a relationship with Hospira.

Correspondence: Timothy D Girard MD MSCI, Division of Allergy, Pulmonary, and Critical Care Medicine, Center for Health Services Research, 6th Floor MCE 6110, Vanderbilt University School of Medicine, Nashville TN 37232-8300. E-mail: timothy.girard@vanderbilt.edu.
}

DOI: $10.4187 /$ respcare. 01708 


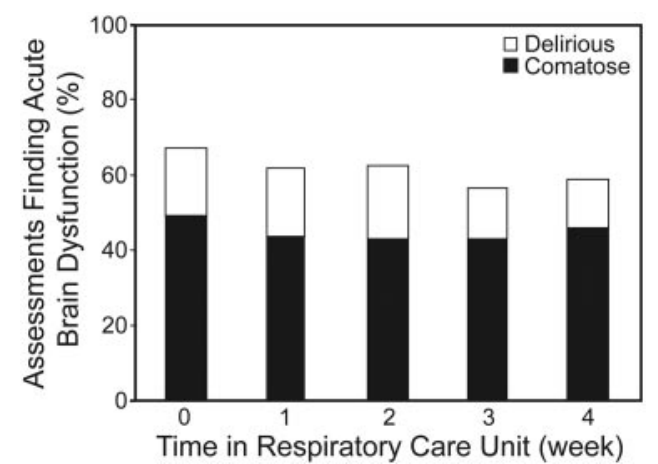

Fig. 1. Prevalence of delirium and coma among 202 patients with chronic critical illness according to week of respiratory care unit stay. (Adapted from Reference 16.)

that 29-69\% of patients experienced brain dysfunction days to weeks into their $\mathrm{CCI}^{16,18}$; the weekly prevalence of acute brain dysfunction in one of these studies is shown in Figure 1. All of the studies examining brain dysfunction in patients with CCI were single-center investigations; differences in severity of illness, use of sedatives, and timing of transfers all may have contributed to the varying rates of brain dysfunction observed. Though large, prospective, multicenter studies are needed to better gauge the general prevalence of coma and delirium during CCI, existing studies clearly demonstrate that brain dysfunction remains a common organ failure after the transition from acute to CCI.

Delirium was considered for decades to be an inconsequential (albeit bothersome) complication of being treated in an ICU, where environmental factors (such as loud noise and lack of sunlight) were believed to cause "ICU psychosis," which resolved prior to ICU discharge and had no lasting effects. Evidence to the contrary has now made it clear that delirium is independently associated with numerous adverse outcomes, including self-extubation and removal of devices, ${ }^{21}$ prolonged ICU ${ }^{22}$ and hospital stays, ${ }^{11,23}$ and increased mortality. ${ }^{5,14,22}$ In a study of 275 mechanically ventilated patients, Ely et $\mathrm{a}^{5}$ found that patients who developed delirium at any time during their ICU stay had significantly higher 6-month mortality than those without delirium, after adjusting for potential confounders such as age, coma, and severity of illness. In addition, duration of delirium was an independent predictor of 6-month mortality, with each additional day of delirium increasing the hazard of dying by $10 \%$. These results have since been duplicated by numerous studies, including 2 that specifically found duration of delirium in the ICU to independently predict 30 -day ${ }^{22}$ and 1-year mortality. ${ }^{14}$

Patients who survive critical illness despite long periods of delirium are at high risk for another adverse neurologic outcome, long-term cognitive impairment. The acute symptoms of delirium, including disturbances in consciousness, resolve for most long-term survivors, but other symptoms of brain dysfunction can persist for months to years. In the

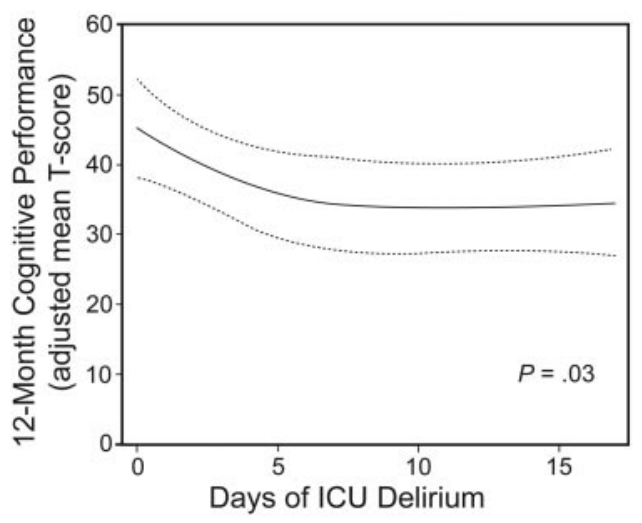

Fig. 2. Relationship between duration of delirium and average cognitive performance measured at 12-month follow-up in 52 patients who survived acute critical illness with respiratory failure. Duration of delirium independently predicted average performance on a battery of 9 neuropsychological tests after adjusting for age, education, preexisting cognitive function, severity of illness, severe sepsis, sedation protocol use, and total benzodiazepine, opiate, and propofol doses administered in the ICU $(P=.03)$. (From Reference 13 , with permission.)

only study to date to examine the relationship between duration of delirium in the ICU and long-term cognitive function, longer periods of delirium were associated with worse scores on a battery of 9 cognitive tests 1 year after the episode of delirium, even after adjusting for potential confounders such as age, severity of illness, and preexisting cognitive impairment (Fig. 2). ${ }^{13}$

Despite a large and growing body of evidence showing that coma and delirium have great prognostic importance during the acute period of critical illness, the prognostic importance of acute brain dysfunction in patients with CCI is less clear. Though Nelson et al ${ }^{16}$ found that more days of delirium during CCI were significantly associated with discharge to a post-acute care facility as well as prolonged hospital stays and poor long-term functional status, the same study found no significant association between delirium and short- or long-term mortality. Similarly, Ceriana et $\mathrm{al}^{18}$ found no correlation between scores on the Intensive Care Delirium Screening Checklist ${ }^{24}$ and mortality among step-down unit patients, the majority of whom had CCI. Finally, Jubran and co-workers ${ }^{19}$ found that transient acute brain dysfunction was actually associated with improved survival in a multivariable analysis of long-term acute care patients. Nearly one third of patients in this study had transient coma and/or delirium, whereas another third had normal mental status, and the final third had persistent coma-delirium; thus, the results regarding transient coma-delirium and survival may primarily reflect the comparison with those patients whose acute brain dysfunction was persistent, a group whose outcomes one would expect to be worse than those with transient delirium.

With only limited data available on the short-term out- 
Brain Dysfunction in Patients with Chronic Critical Illness

comes associated with delirium during $\mathrm{CCI}$ and no data available on long-term outcomes, firm conclusions cannot yet be made regarding the prognostic importance of delirium in this patient population. But, with preliminary data suggesting that delirium often persists for more than a few days during CCI, ${ }^{16}$ and numerous ICU studies showing that duration of delirium predicts adverse long-term outcomes, $5,13,14,22$ it is reasonable to hypothesize that chronically critically ill patients with prolonged delirium are at high risk for poor long-term outcomes, especially longterm brain dysfunction; this hypothesis can and should be tested in future studies.

\section{Long-Term Brain Dysfunction in Survivors of Critical Illness}

Survivors of acute critical illness usually experience resolution of coma and delirium as their acute illness improves. Despite this evidence of some neurologic improvement, brain dysfunction often persists, taking on a different form. Over a dozen studies have demonstrated that longterm cognitive impairment affects between $20 \%$ and $80 \%$ of ICU survivors, depending on the population studied and the methods used to identify impairment. Hopkins et al, ${ }^{25}$ for example, examined 55 patients 1 year after mechanical ventilation for ARDS and found that $78 \%$ were impaired in one or more cognitive domains such as memory, concentration, attention, and processing speed. The majority of studies, in fact, have found that memory is impaired in many patients for months to years after a critical illness; the other domain of cognition most commonly impaired is executive function. ${ }^{26}$ The cognitive deficits identified in these studies adversely affect patients in numerous ways, making it difficult or impossible to return to work, and reducing quality of life. Rothenhausler and colleagues 27 examined 46 ARDS survivors an average of 6 years after their illness and found that the 11 (24\%) with cognitive impairment were unable to return to work and had poor health-related quality of life, which was independently associated with long-term cognitive impairment.

The rigorous methods used to assess cognitive function in studies of patients who survived acute critical illnesswhich included prospective examination of patients with comprehensive neuropsychological batteries- have not yet been applied to study survivors of CCI, but 4 investigations have identified long-term brain dysfunction in this population using other methods (Table 2). In these studies, which examined patients 3 months to $2+$ years after CCI, $40-77 \%$ of patients exhibited signs of long-term brain dysfunction, manifest as poor Mini-Mental State Examination scores or inability to respond to a telephone interview. The specific nature and severity of the long-term cognitive deficits that affect survivors of CCI are not known, but it is likely that the domains of cognition that
Table 2. Prevalence of Long-Term Brain Dysfunction in Survivors of Chronic Critical Illness

\begin{tabular}{lllrc}
\hline \hline First Author & Year & \multicolumn{1}{c}{ Population } & $N$ & $\begin{array}{c}\text { Brain } \\
\text { Dysfunction, } \\
(\%)\end{array}$ \\
\hline Rabinstein $^{28}$ & 2004 & Stroke with tracheotomy & 97 & $74 *$ \\
Nelson $^{16}$ & 2006 & RCU & 98 & $77 \dagger$ \\
& & & 85 & $71 \%$ \\
Hung $^{29}$ & 2010 & PMV & 142 & $61 \S$ \\
Unroe $^{30}$ & 2010 & PMV & 90 & $40 \|$ \\
& & & 70 & $44 \mathbb{I}$
\end{tabular}

\footnotetext{
* Glasgow Outcome Scale score 1-3 one year after stroke.

$\dagger$ Unable to respond to telephone interview conducted 3 months after discharge.

¥ Unable to respond to telephone interview conducted 12 months after discharge.

$\S$ Mini-Mental State Examination score $<15$ on interview conducted an average \pm SD of

$19.1 \pm 26.0$ months into the ventilator stay.

$\|$ Mini-Mental State Examination score $<20$ on interview conducted 3 months after discharge.

II Mini-Mental State Examination score $<20$ on interview conducted 12 months after

discharge.

PMV $=$ prolonged mechanical ventilation $(\geq 21$ days with tracheostomy or $\geq 4$ days with tracheostomy)

RCU $=$ respiratory care unit $(100 \%$ mechanically ventilated $)$
}

are impaired in this population are similar to those observed in investigations of survivors of acute critical illness. The preliminary data from studies of CCI survivors suggest that long-term cognitive impairment in this population is often severe; a Mini-Mental State Examination score $<15$, the cutoff used in one study, represents a cognitive state similar to that observed in patients with moderate dementia. Detailed studies are needed to fully elucidate the prevalence and character of long-term cognitive impairment among survivors of CCI.

Despite the high prevalence and adverse effects of longterm cognitive impairment in survivors of both acute and chronic critical illness, this complication is often overlooked by clinicians and misunderstood by patients and their caregivers. In a study of ARDS patients, Hopkins and co-workers ${ }^{31}$ found that $46(70 \%)$ of 66 survivors were cognitively impaired at hospital discharge, yet only 9 patients $(<20 \%$ of those who were impaired) were subsequently identified as having cognitive impairment by clinicians caring for these patients. Not only are these deficits often unaddressed by clinicians during the long period of recovery from critical illness, but the potential for poor cognitive outcomes is frequently not discussed with critically ill patients or their families. Nelson et al ${ }^{32}$ studied patients with CCI to determine the informational needs of patients and their families. Among 96 surrogates asked if they received information about "what the patient's cognitive status (ability to think and understand, mental state) is expected to be when he leaves the hospital," only 33 (34\%) reported receiving any information. In contrast, 99\% of respondents rated information about long-term cognitive outcomes as important to decision making, a result in keeping with the findings of an earlier study by Fried and 
colleagues. ${ }^{33}$ They surveyed 226 seriously ill patients to determine outcomes considered important when determining treatment preferences; cognitive impairment was reported by these patients to be a less acceptable outcome than death. Thus, though it remains unclear how best to identify patients at highest risk for long-term cognitive impairment after CCI, the very real possibility of such an outcome should be acknowledged by clinicians and discussed openly with patients and their surrogates.

\section{Management of Brain Dysfunction During Critical Illness}

The management of brain dysfunction during and after CCI has not been specifically examined, but a growing body of literature on brain dysfunction during the period of acute critical illness points to a number of treatment strategies that may be of import for the population with CCI. As with any patient, an acute change in mental status (whether new-onset delirium or coma) during CCI should prompt an evaluation for an etiology and/or modifiable risk factors. Signs of stroke or seizure accompanying newonset coma, for example, should prompt directed evaluations with neuroimaging or electroencephalography, whereas newly delirious patients should be evaluated for one or more of the numerous modifiable risk factors for delirium identified in ICU studies, including infection/sepsis, hypotension, hypoxemia, hyponatremia, hypocalcemia, azotemia, and exposure to sedating medications. ${ }^{34-36}$ The identification of such a risk factor in the setting of newonset delirium may afford the clinician an opportunity to intervene, since treating the risk factor may interrupt the process of brain dysfunction.

Though placement of tracheostomy - a nearly universal treatment for patients with CCI-may lead to less sedative use, ${ }^{37}$ exposure to sedating medications remains common during CCI and represents a potential risk factor for brain dysfunction in this vulnerable population. Nelson et al ${ }^{16}$ reported that $128(63 \%)$ of 203 respiratory care unit patients received benzodiazepines during CCI, and 160 (79\%) received opioid analgesics. Though analyses of sedative doses and acute brain dysfunction in this study found no associations, benzodiazepine exposure has consistently been associated with delirium in ICU studies. Pandharipande and colleagues, ${ }^{36}$ for example, found that lorazepam dose independently predicted the probability of delirium in a study of mechanically ventilated medical ICU patients; those treated with higher doses of lorazepam on a given ICU day were more likely to be delirious the following day than patients who received small doses $(<3 \mathrm{mg})$ or no lorazepam. The median benzodiazepine dose used in the respiratory care unit studied by Nelson et al ${ }^{16}$ was $1 \mathrm{mg} / \mathrm{d}$ in lorazepam equivalents, which may explain the lack of an association with delirium in that study. Those patients with CCI who receive higher doses of benzodiazepines are likely at increased risk for delirium, as this association has been observed in medical, ${ }^{36,39}$ surgical,,${ }^{40}$ mixed, ${ }^{21}$ trauma,${ }^{40}$ and burn ${ }^{41}$ ICU patients who are moderately or heavily sedated with benzodiazepines. Thus, benzodiazepines should be avoided, whenever possible, during the treatment of patients with CCI.

When sedation is needed to manage patients with CCI, alternatives to benzodiazepines include opioids, which can provide analgesia and sedation, as well as propofol, dexmedetomidine, and antipsychotic medications. None of these agents have been studied specifically in the setting of CCI, so results from studies during acute critical illness should be extrapolated with some caution. Signs of agitation, anxiety, or discomfort during CCI should always prompt clinicians to consider whether opioids are needed, since pain is common during CCI; two thirds of patients reported pain in one study of $\mathrm{CCI}$, with nearly half of all patients reporting moderate to severe pain. ${ }^{42}$ The use of opioids in this setting will not only control pain but may also reduce the risk of delirium. ${ }^{43}$

With its rapid onset of action, propofol is an attractive option for the treatment of acute agitation in patients who have protected airways, especially for severe agitation that requires immediate resolution. Compared head-to-head with benzodiazepines in several randomized trials, propofol provided more accurate sedation (ie, less oversedation) and led to shorter ventilator time. ${ }^{44-46}$ Time-limited courses of propofol sedation, in contrast to longer-term continuous sedation, have been used effectively in the ICU and should be considered when managing severe agitation during CCI. Strøm et $\mathrm{al}^{47}$ included 6-hour courses of propofol in a protocol that emphasized "no sedation," prioritizing asneeded morphine and haloperidol over continuous sedation in the management of mechanically ventilated ICU patients; the no sedation protocol reduced time on the ventilator, in the ICU, and in the hospital, compared with standard of care. Similar protocols should be developed to specifically address the needs of patients with CCI, many of whom develop agitation ${ }^{48}$ or other symptoms prompting treatment with sedatives; until such protocols have been tested in this unique patient population, protocols that have been rigorously studied and found to be beneficial among patients with acute critical illness should be considered for the management of patients with CCI.4,49,50

Dexmedetomidine, another alternative to benzodiazepine sedatives, has been studied recently during acute critical illness and may offer advantages in the management of patients with CCI. Whereas propofol, similar to the benzodiazepines, potentiates $\gamma$-aminobutyric acid type A $\left(\mathrm{GABA}_{\mathrm{A}}\right)$ receptor activity to produce sedation, dexmedetomidine sedates via stimulation of central $\alpha_{2}$-adrenergic receptors. This GABA-sparing mechanism of action may explain the results of 2 randomized, double-blind, multi- 
center trials that found that mechanically ventilated ICU patients who were sedated with dexmedetomidine had less delirium on a daily basis than those sedated with a benzodiazepine. ${ }^{10,38}$ Dexmedetomidine was also compared with haloperidol for the treatment of agitated delirium during acute critical illness; in a small randomized trial, ${ }^{51}$ those treated with dexmedetomidine were extubated and discharged from the ICU more quickly than patients treated with haloperidol, suggesting dexmedetomidine may be effective both for sedation and possibly for treatment of agitated delirium, though dexmedetomidine for this indication needs to be studied in larger clinical trials.

Haloperidol remains the most widely used medication for the treatment of delirium during critical illness, with atypical antipsychotics growing in popularity. Nearly $40 \%$ of respondents in a recent international survey, for example, reported using atypical antipsychotics to manage delirium, whereas almost $90 \%$ reported using haloperidol for this indication. ${ }^{52}$ Similarly, Nelson et al ${ }^{16}$ found that two thirds of patients with CCI received antipsychotics during their respiratory care unit stay. The use of this class of medications for the treatment of delirium during both acute and chronic critical illness is supported primarily by results of studies conducted outside the ICU. Hu et al ${ }^{53}$ studied haloperidol and olanzapine in a randomized, placebo-controlled trial of older patients with delirium and found that both the typical and the atypical antipsychotic resulted in more rapid resolution of delirium than did placebo. Similarly, Kalisvaart and co-workers ${ }^{54}$ found that haloperidol treatment prior to hip surgery led to a shorter period of postoperative delirium, compared with placebo. Only 2 placebo-controlled trials, both pilot in nature, have examined the effects of antipsychotics among patients with acute critical illness. In one study, our research team found no difference in duration of acute brain dysfunction among mechanically ventilated ICU patients treated with haloperidol versus ziprasidone versus placebo. ${ }^{55}$ Alternatively, in a study of delirious ICU patients who uniformly failed to respond to haloperidol, Devlin et a ${ }^{56}$ found that addition of quetiapine rather than placebo resulted in earlier resolution of delirium (Fig. 3). With the only 2 placebocontrolled trials of antipsychotics for delirium in the ICU being very small and giving conflicting results, it is impossible to know at this time whether these medications are efficacious for delirium during critical illness. We are therefore coordinating a large, multicenter, phase 3, randomized controlled trial ${ }^{57}$ to answer this question, but future research will be needed specifically to examine the effect of antipsychotics during CCI. Until such data are available, it is likely that antipsychotics will continue to be recommended for the treatment of delirium during critical illness, whether acute or chronic. 58

The management of brain dysfunction during critical illness is not limited to pharmacologic interventions.

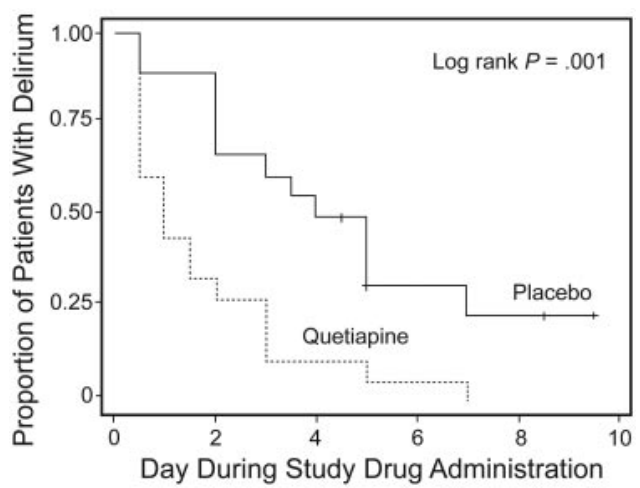

Fig. 3. Effect of treatment with quetiapine versus placebo on resolution of delirium in 36 patients with acute critical illness. Both groups of patients were treated using the same as-needed intravenous haloperidol protocol. Compared with placebo, the addition of quetiapine resulted in faster resolution of delirium $(P=.001)$. (From Reference 56, with permission.)

Though not yet studied in an ICU population, several multicomponent, nonpharmacologic strategies have been found to reduce delirium incidence among acutely ill older patients. ${ }^{59}$ Similar approaches are likely to be of benefit for patients with CCI, and interventions such as orientation, nonpharmacologic sleep protocols, and vision/hearing aids are generally safe and require little in the way of resources. A nonpharmacologic intervention that can, in contrast, be very resource intense-early physical and occupational therapy (often referred to as early mobility) has recently been examined in several different ICU populations. In a study of 103 respiratory ICU patients, a population that included many CCI patients, Bailey and colleagues $^{60}$ showed that early mobility was safe and feasible. Over two thirds of the patients, in fact, were able to ambulate $>100$ feet at the time of ICU discharge. Subsequently, multiple interventional studies, both randomized $^{61}$ and non-randomized, ${ }^{62,63}$ have examined early physical/occupational/mobility therapy during acute critical illness and found these interventions to improve numerous outcomes, such as return to functional independence and hospital stay. Two of these trials specifically assessed patients for delirium and found that early physical/occupational therapy reduced the duration of delirium during critical illness, ${ }^{61,63}$ suggesting that early physical activity - an important component of delirium prevention programs previously studied outside the ICU—might significantly reduce brain dysfunction during acute and chronic critical illness.

A complementary approach to the management of brain dysfunction in the midst of critical illness is the rehabilitation of cognitive function after critical illness. Though a large body of literature supports the use of cognitive rehabilitation in other patient populations, such as those with traumatic brain injury, this intervention has only recently been studied among survivors of critical illness. In a small, 
pilot investigation, Jackson and co-workers ${ }^{64}$ randomized patients being discharged from the hospital after acute critical illness to usual care or a multicomponent rehabilitation program combining cognitive, physical, and functional training. Three months later, those managed with the rehabilitation program had better cognitive and functional outcomes than those in the control group, a finding that should prompt further investigation in large, multicenter trails of cognitive rehabilitation after critical illness.

\section{Future Directions}

A substantial body of literature has grown over the past decade to inform critical care practitioners about the prevalence, outcomes, and management of acute and long-term brain dysfunction attributable to critical illness. The large majority of these investigations, however, have been limited to patients with acute critical illness. Therefore, targeted studies are needed to elucidate the effects of CCI on the brain and to identify therapies directed at brain dysfunction during CCI that are safe and efficacious. Specifically, multicenter studies with a broad array of chronically critically ill patients are needed to examine the prevalence and outcomes of coma and delirium in this population as well as long-term brain dysfunction in survivors of CCI. Risk factors for and pathophysiologic mechanisms of acute and long-term brain dysfunction due to CCI should also be thoroughly illuminated. The latter, mechanisms of brain dysfunction, have only recently been studied during acute critical illness and only in small, hypothesis-generating studies. Being preliminary in nature, the findings of these studies are not reviewed here, but these results and those of recent animal studies should guide investigations of the mechanisms of brain dysfunction during CCI. Lastly, though interventions that effectively reduce brain dysfunction during acute critical illness should be implemented among those with CCI until more data are forthcoming, randomized trials are needed to determine whether interventions used during acute critical illness are effective in CCI. In addition, novel interventions that may be of particular benefit to patients with CCI should be sought and studied in this setting.

\section{Summary}

Acute brain dysfunction, in the form of coma and delirium, occurs frequently during both acute and chronic critical illness, and patients who survive either form of critical illness remain at high risk for ongoing long-term brain dysfunction. A growing number of studies have found that coma, delirium, and long-term brain dysfunction are associated with numerous adverse outcomes among acute critical illness patients, including prolonged hospitalizations, increased healthcare costs, and increased mortality. Though not yet well studied in the setting of CCI, brain dysfunc- tion may be associated with adverse outcomes for these vulnerable patients. Thus, interventions to prevent and/or treat acute and long-term brain dysfunction should be implemented in the care of patients with CCI. Multicenter randomized trials are needed to determine which interventions are most effective for such patients, and until these data are available, management strategies that have been proven beneficial during acute critical illness-such as reduction of sedative exposure, especially to benzodiazepines, and early physical and occupational therapy - should be employed during the treatment of patients with CCI.

\section{REFERENCES}

1. Posner JB, Saper CB, Schiff N, Plum F. Plum and Posner's diagnosis of stupor and coma. New York: Oxford University Press; 2007.

2. Diagnostic and statistical manual of mental disorders, fourth edition, Washington, DC: American Psychiatric Association; 2000.

3. Ely EW, Truman B, Shintani A, Thomason JW, Wheeler AP, Gordon $\mathrm{S}$, et al. Monitoring sedation status over time in ICU patients: reliability and validity of the Richmond Agitation-Sedation Scale (RASS). JAMA 2003;289(22):2983-2991.

4. Girard TD, Kress JP, Fuchs BD, Thomason JW, Schweickert WD, Pun BT, et al. Efficacy and safety of a paired sedation and ventilator weaning protocol for mechanically ventilated patients in intensive care (Awakening and Breathing Controlled trial): a randomised controlled trial. Lancet 2008;371(9607):126-134.

5. Ely EW, Shintani A, Truman B, Speroff T, Gordon SM, Harrell FE, Jr., et al. Delirium as a predictor of mortality in mechanically ventilated patients in the intensive care unit. JAMA 2004;291(14):17531762.

6. Hamel MB, Goldman L, Teno J, Lynn J, Davis RB, Harrell FE Jr, et al. Identification of comatose patients at high risk for death or severe disability. SUPPORT Investigators. Understand Prognoses and Preferences for Outcomes and Risks of Treatments. JAMA 1995; 273(23):1842-1848.

7. Ely EW, Inouye SK, Bernard GR, Gordon S, Francis J, May L, et al. Delirium in mechanically ventilated patients: validity and reliability of the confusion assessment method for the intensive care unit (CAM-ICU). JAMA 2001;286(21):2703-2710.

8. Micek ST, Anand NJ, Laible BR, Shannon WD, Kollef MH. Delirium as detected by the CAM-ICU predicts restraint use among mechanically ventilated medical patients. Crit Care Med 2005;33(6): 1260-1265.

9. Pandharipande P, Cotton BA, Shintani A, Thompson J, Costabile S, Truman Pun B, et al. Motoric subtypes of delirium in mechanically ventilated surgical and trauma intensive care unit patients. Intensive Care Med 2007;33(10):1726-1731.

10. Riker RR, Shehabi Y, Bokesch PM, Ceraso D, Wisemandle W, Koura F, et al. Dexmedetomidine vs midazolam for sedation of critically ill patients: a randomized trial. JAMA 2009;301(5):489-499.

11. Thomason JW, Shintani A, Peterson JF, Pun BT, Jackson JC, Ely EW. Intensive care unit delirium is an independent predictor of longer hospital stay: a prospective analysis of 261 non-ventilated patients. Crit Care 2005;9(4):R375-R381.

12. Luetz A, Heymann A, Radtke FM, Chenitir C, Neuhaus U, Nachtigall I, et al. Different assessment tools for intensive care unit delirium: which score to use? Crit Care Med 2010;38(2):409-418.

13. Girard TD, Jackson JC, Pandharipande PP, Pun BT, Thompson JL, Shintani AK, et al. Delirium as a predictor of long-term cognitive impairment in survivors of critical illness. Crit Care Med 2010;38(7): 1513-1520. 


\section{Brain Dysfunction in Patients with Chronic Critical Illness}

14. Pisani MA, Kong SY, Kasl SV, Murphy TE, Araujo KL, Van Ness PH. Days of delirium are associated with 1-year mortality in an older intensive care unit population. Am J Respir Crit Care Med 2009; 180(11):1092-1097.

15. Weinert CR. Epidemiology of psychiatric medication use in patients recovering from critical illness at a long-term acute-care facility. Chest 2001;119(2):547-553.

16. Nelson JE, Tandon N, Mercado AF, Camhi SL, Ely EW, Morrison RS. Brain dysfunction: another burden for the chronically critically ill. Arch Intern Med 2006;166(18):1993-1999.

17. Scheinhorn DJ, Hassenpflug MS, Votto JJ, Chao DC, Epstein SK, Doig GS, et al. Ventilator-dependent survivors of catastrophic illness transferred to 23 long-term care hospitals for weaning from prolonged mechanical ventilation. Chest 2007;131(1):76-84.

18. Ceriana P, Fanfulla F, Mazzacane F, Santoro C, Nava S. Delirium in patients admitted to a step-down unit: analysis of incidence and risk factors. J Crit Care 2010;25(1):136-143.

19. Jubran A, Lawm G, Kelly J, Duffner LA, Gungor G, Collins EG, et al. Depressive disorders during weaning from prolonged mechanical ventilation. Intensive Care Med 2010;36(5):828-835.

20. Ouimet S, Riker R, Bergeron N, Cossette M, Kavanagh B, Skrobik Y. Subsyndromal delirium in the ICU: evidence for a disease spectrum. Intensive Care Med 2007;33(6):1007-1013.

21. Ouimet S, Kavanagh BP, Gottfried SB, Skrobik Y. Incidence, risk factors and consequences of ICU delirium. Intensive Care Med 2007; 33(1):66-73

22. Shehabi Y, Riker RR, Bokesch PM, Wisemandle W, Shintani A, Ely EW. Delirium duration and mortality in lightly sedated, mechanically ventilated intensive care patients. Crit Care Med 2010;38(12): 2311-2318.

23. Ely EW, Gautam S, Margolin R, Francis J, May L, Speroff T, et al. The impact of delirium in the intensive care unit on hospital length of stay. Intensive Care Med 2001;27(12):1892-1900.

24. Bergeron N, Dubois MJ, Dumont M, Dial S, Skrobik Y. Intensive Care Delirium Screening Checklist: evaluation of a new screening tool. Intensive Care Med 2001;27(5):859-864.

25. Hopkins RO, Weaver LK, Pope D, Orme JF, Bigler ED, Larson LV. Neuropsychological sequelae and impaired health status in survivors of severe acute respiratory distress syndrome. Am J Respir Crit Care Med 1999;160(1):50-56.

26. Hopkins RO, Jackson JC. Long-term neurocognitive function after critical illness. Chest 2006;130(3):869-878.

27. Rothenhausler HB, Ehrentraut S, Stoll C, Schelling G, Kapfhammer HP. The relationship between cognitive performance and employment and health status in long-term survivors of the acute respiratory distress syndrome: results of an exploratory study. Gen Hosp Psychiatry 2001;23(2):90-96.

28. Rabinstein AA, Wijdicks EF. Outcome of survivors of acute stroke who require prolonged ventilatory assistance and tracheostomy. Cerebrovasc Dis 2004;18(4):325-331.

29. Hung MC, Yan YH, Fan PS, Lin MS, Chen CR, Kuo LC, et al. Measurement of quality of life using EQ-5D in patients on prolonged mechanical ventilation: comparison of patients, family caregivers, and nurses. Qual Life Res 2010;19(5):721-727.

30. Unroe M, Kahn JM, Carson SS, Govert JA, Martinu T, Sathy SJ, et al. One-year trajectories of care and resource utilization for recipients of prolonged mechanical ventilation: a cohort study. Ann Intern Med 2010;153(3):167-175.

31. Hopkins RO, Weaver LK, Collingridge D, Parkinson RB, Chan KJ, Orme JF Jr. Two-year cognitive, emotional, and quality-of-life outcomes in acute respiratory distress syndrome. Am J Respir Crit Care Med 2005;171(4):340-347.
32. Nelson JE, Mercado AF, Camhi SL, Tandon N, Wallenstein S, August GI, et al. Communication about chronic critical illness. Arch Intern Med 2007;167(22):2509-2515.

33. Fried TR, Bradley EH, Towle VR, Allore H. Understanding the treatment preferences of seriously ill patients. N Engl J Med 2002; 346(14):1061-1066.

34. McNicoll L, Pisani MA, Zhang Y, Ely EW, Siegel MD, Inouye SK. Delirium in the intensive care unit: occurrence and clinical course in older patients. J Am Geriatr Soc 2003;51(5):591-598.

35. Aldemir M, Ozen S, Kara IH, Sir A, Bac B. Predisposing factors for delirium in the surgical intensive care unit. Crit Care 2001;5(5): 265-270.

36. Pandharipande P, Shintani A, Peterson J, Pun BT, Wilkinson GR, Dittus RS, et al. Lorazepam is an independent risk factor for transitioning to delirium in intensive care unit patients. Anesthesiology 2006;104(1):21-26.

37. Nieszkowska A, Combes A, Luyt CE, Ksibi H, Trouillet JL, Gibert $\mathrm{C}$, et al. Impact of tracheotomy on sedative administration, sedation level, and comfort of mechanically ventilated intensive care unit patients. Crit Care Med 2005;33(11):2527-2533.

38. Pandharipande PP, Pun BT, Herr DL, Maze M, Girard TD, Miller $\mathrm{RR}$, et al. Effect of sedation with dexmedetomidine vs lorazepam on acute brain dysfunction in mechanically ventilated patients: the MENDS randomized controlled trial. JAMA 2007;298(22):2644-2653.

39. Pisani MA, Murphy TE, Araujo KL, Slattum P, Van Ness PH, Inouye SK. Benzodiazepine and opioid use and the duration of intensive care unit delirium in an older population. Crit Care Med 2009; 37(1):177-183.

40. Pandharipande P, Cotton BA, Shintani A, Thompson J, Pun BT, Morris JA Jr, et al. Prevalence and risk factors for development of delirium in surgical and trauma intensive care unit patients. J Trauma 2008;65(1):34-41.

41. Agarwal V, O'Neill PJ, Cotton BA, Pun BT, Haney S, Thompson J, et al. Prevalence and risk factors for development of delirium in burn intensive care unit patients. J Burn Care Res 2010;31(5):706-715.

42. Nelson JE, Meier DE, Litke A, Natale DA, Siegel RE, Morrison RS. The symptom burden of chronic critical illness. Crit Care Med 2004; 32(7):1527-1534.

43. Morrison RS, Magaziner J, Gilbert M, Koval KJ, McLaughlin MA, Orosz G, et al. Relationship between pain and opioid analgesics on the development of delirium following hip fracture. J Gerontol A Biol Sci Med Sci 2003;58(1):76-81.

44. Hall RI, Sandham D, Cardinal P, Tweeddale M, Moher D, Wang X, et al. Propofol vs midazolam for ICU sedation: a Canadian multicenter randomized trial. Chest 2001;119(4):1151-1159.

45. Carson SS, Kress JP, Rodgers JE, Vinayak A, Campbell-Bright S, Levitt $\mathrm{J}$, et al. A randomized trial of intermittent lorazepam versus propofol with daily interruption in mechanically ventilated patients. Crit Care Med 2006;34(5):1326-1332.

46. Barrientos-Vega R, Mar Sanchez-Soria M, Morales-Garcia C, Robas-Gomez A, Cuena-Boy R, Ayensa-Rincon A. Prolonged sedation of critically ill patients with midazolam or propofol: impact on weaning and costs. Crit Care Med 1997;25(1):33-40.

47. Strøm T, Martinussen T, Toft P. A protocol of no sedation for critically ill patients receiving mechanical ventilation: a randomised trial. Lancet 2010;375(9713):475-480.

48. Estenssoro E, Reina R, Canales HS, Saenz MG, Gonzalez FE, Aprea $\mathrm{MM}$, et al. The distinct clinical profile of chronically critically ill patients: a cohort study. Crit Care 2006;10(3):R89.

49. Brook AD, Ahrens TS, Schaiff R, Prentice D, Sherman G, Shannon $\mathrm{W}$, et al. Effect of a nursing-implemented sedation protocol on the duration of mechanical ventilation. Crit Care Med 1999;27(12): 2609-2615. 


\section{Brain Dysfunction in Patients with Chronic Critical Illness}

50. Kress JP, Pohlman AS, O'Connor MF, Hall JB. Daily interruption of sedative infusions in critically ill patients undergoing mechanical ventilation. N Engl J Med 2000;342(20):1471-1477.

51. Reade MC, O'Sullivan K, Bates S, Goldsmith D, Ainslie WR, Bellomo R. Dexmedetomidine vs. haloperidol in delirious, agitated, intubated patients: a randomised open-label trial. Crit Care 2009;13(3): R75.

52. Patel RP, Gambrell M, Speroff T, Scott TA, Pun BT, Okahashi J, et al. Delirium and sedation in the intensive care unit: survey of behaviors and attitudes of 1384 healthcare professionals. Crit Care Med 2009;37(3):825-832.

53. Hu H, Deng W, Yang H. A prospective random control study comparison of olanzapine and haloperidol in senile delirium. Chongqing Med J 2004;8:1234-1237.

54. Kalisvaart KJ, de Jonghe JF, Bogaards MJ, Vreeswijk R, Egberts TC, Burger BJ, et al. Haloperidol prophylaxis for elderly hip-surgery patients at risk for delirium: a randomized placebo-controlled study. J Am Geriatr Soc 2005;53(10):1658-1666.

55. Girard TD, Pandharipande PP, Carson SS, Schmidt GA, Wright PE, Canonico AE, et al. Feasibility, efficacy, and safety of antipsychotics for intensive care unit delirium: the MIND randomized, placebocontrolled trial. Crit Care Med 2010;38(2):428-437.

56. Devlin JW, Roberts RJ, Fong JJ, Skrobik Y, Riker RR, Hill NS, et al. Efficacy and safety of quetiapine in critically ill patients with delirium: a prospective, multicenter, randomized, doubleblind, placebo-controlled pilot study. Crit Care Med 2010;38(2): 419-427.

57. The modifying the impact of ICU-associated neurological dysfunc-
tion-USA (MIND-USA) study. http://clinicaltrials.gov/ct2/show/ NCT01211522. Accessed November 2, 2011.

58. Jacobi J, Fraser GL, Coursin DB, Riker RR, Fontaine D, Wittbrodt ET, et al. Clinical practice guidelines for the sustained use of sedatives and analgesics in the critically ill adult. Crit Care Med 2002; 30(1):119-141.

59. Inouye SK, Bogardus ST Jr, Charpentier PA, Leo-Summers L, Acampora D, Holford TR, et al. A multicomponent intervention to prevent delirium in hospitalized older patients. N Engl J Med 1999; 340(9):669-676.

60. Bailey P, Thomsen GE, Spuhler VJ, Blair R, Jewkes J, Bezdjian L, et al. Early activity is feasible and safe in respiratory failure patients. Crit Care Med 2007;35(1):139-145.

61. Schweickert WD, Pohlman MC, Pohlman AS, Nigos C, Pawlik AJ, Esbrook CL, et al. Early physical and occupational therapy in mechanically ventilated, critically ill patients: a randomised controlled trial. Lancet 2009;373(9678):1874-1882.

62. Morris PE, Goad A, Thompson C, Taylor K, Harry B, Passmore L, et al. Early intensive care unit mobility therapy in the treatment of acute respiratory failure. Crit Care Med 2008;36(8):2238-2243.

63. Needham DM, Korupolu R, Zanni JM, Pradhan P, Colantuoni E, Palmer JB, et al. Early physical medicine and rehabilitation for patients with acute respiratory failure: a quality improvement project. Arch Phys Med Rehabil 2010;91(4):536-542.

64. Jackson JC, Ely EW, Morey MC, Anderson VM, Siebert CS, Denne LB, et al. Cognitive and physical rehabilitation of intensive care unit survivors: results of the RETURN randomized controlled pilot investigation. Crit Care Med 2012;40(4):1088-1097.

\section{Discussion}

White: What proportion of decreased cognitive function can you actually attribute to the ICU experience? Without before and after data, you don't know what their cognitive function was before they entered the study, because you can't get that. Are there data on patients of similar age and function where they could figure out what percent of the decrement in cognitive function was attributable to the CCI and how much of it was that's just the way they were?

Girard: The inability to directly assess pre-ICU cognitive function has, in many ways, been the Achilles heel in this area of research. You can't, in most cases, identify patients and test them prior to critical illness, because an ICU stay is not like a surgery that is planned. Instead, it usually happens unexpectedly. But in the last couple of years there have fortunately been some very elegant studies that have addressed this challenge by making use of long, prospective cohort studies involving older patients who were being assessed at regular intervals for cognitive impairment. When some of these patients developed critical illness, the opportunity arose to compare preand post-ICU cognitive function.

In one of these studies, Jack Iwashyna and his colleagues ${ }^{1}$ used the Health and Retirement Study cohort and found that patients who had been tested before and after episodes of severe sepsis requiring ICU admission had significantly higher rates of new cognitive impairment. Unlike most studies of ICU survivors, these were patients who were tested before and after severe sepsis, and the risk of developing new cognitive impairment after critical illness was significantly increased, compared with patients who did not develop severe sepsis.

Bill Ehlenbach and his colleagues ${ }^{2}$ analyzed patients in a similar cohort study conducted in Seattle. They studied the effect of acute hospitalizations and ICU stays among patients who had been tested prior to and after those events. They found a very similar result. The rate of cognitive impairment went up significantly for those patients who had a hospitalization or an ICU stay, compared with those who did not.

1. Iwashyna TJ, Ely EW, Smith DM, Langa KM. Long-term cognitive impairment and function disability among survivors of severe sepsis. JAMA 2010;304(16):1787-1794

2. Ehlenbach WJ, Hough CL, Crane PK, Haneuse SJ, Carson SS, Curtis JR, Larson EB. Association between acute care and critical illness hospitalization and cognitive function in older adults. JAMA 2010;303(8):763-770. 
MacIntyre: Can you address sleep deprivation as a cause of delirium? Are there strategies to improve sleep to perhaps reduce that effect?

Girard: We don't have many data on that yet. There is certainly a widely held belief that sleep deprivation can contribute to acute cognitive abnormalities like delirium, and there are many studies of sleep deprivationnot in ICU patients but in other settings, such as among healthy subjects-showing it can lead to acute cognitive deficits. So we have a good sense that sleep deprivation is a problem cognitively, and we also have a pretty good idea that it's a problem during critical illness.

But it's been hard to determine what proportion of the delirium that occurs during critical illness can be attributed to sleep deprivation. In fact, we usually have a hard time attributing delirium to any one specific risk factor or etiology. In one study the average number of risk factors for delirium identified in a population of medical ICU patients was 11 . We'd like to be able to pinpoint whether it was sleep deprivation, their acute infection, their exposure to medications, but we don't know.

Sleep is especially challenging to study in the ICU, because when you study critically ill patients with EEG [electroencephalography] or PSG [polysomnography], which we've done at Vanderbilt, what you find is something that doesn't even fit into the standard classifications of sleep. You can't sit down with an EEG from a mechanically ventilated ICU patient and easily say they've spent this much time in slow-wave sleep. It's a really challenging area of research.

MacIntyre: The reason I brought it up is because it is something we can modify. This practice of doing daily chest X-rays at 3:00 Am and daily blood draws at 4:00 AM and daily vital signs at 5:00 AM: maybe we could rearrange our schedule to better fit theirs.
Girard: I agree completely. We've alluded multiple times today to reducing sedation. Our studies with EEG found that patients who were receiving very high doses of benzodiazepines, which are now infrequently used in our ICU, had very little slow-wave sleep. On physical exam, these patients were comatose, so the ICU team often said the patients were "resting" or "asleep," but they are not sleeping. When you look at the EEGs, the benzodiazepines appeared to be leading to the least amount of slow-wave of any of the patients we studied.

Fan: There may be data forthcoming. Johns Hopkins has launched a sleep quality improvement project, and they are using a staged intervention to improve environmental and pharmacologic alterations to sleep in ICU patients. They're collecting sleep questionnaires and monitoring delirium and doing some simple neurocognitive testing. So there may be some more information coming on sleep quality and ICU delirium. Tim, did that study by Jim Jackson ${ }^{1}$ specifically look at physical rehabilitation? Do you have comments on the role of neurocognitive rehabilitation in the ICU, like getting patients in the ICU to play Scrabble or Sudoku?

1. Jackson JC, Ely EW, Morey MC, Anderson VM, Denne LB, Clune J, et al. Cognitive and physical rehabilitation of intensive care unit survivors: results of the RETURN randomized controlled pilot investigation. Crit Care Med 2012;40(4):1088-1097.

Girard: That study examined a combined cognitive and physical rehabilitation program that was started after hospital discharge. The intervention group received in-home physical therapy via either a physical therapist who went to the patient's home or a telecommunications system that was brought to the patient's home and set up so that the physical therapist could remotely direct the therapy. They also received a specific type of cognitive rehabilitation, goal management train- ing, that's shown significant benefit in other patient populations.

Regarding other potential interventions, such as having them do cognitively stimulating activities, we're currently doing a pilot study called ACTICU (Activity and Cognitive Therapy in the ICU) to determine whether an intervention that combines cognitive and physical therapy very early during critical illness is feasible and safe. As soon as the patient passes some basic safety criteria, very early in the course of their illness, we're doing physical and cognitive therapy that involves many components, including games and other stimulating activities.

Muldoon:* You showed a slide that said about $50 \%$ of delirium is missed, and another slide that showed that, for those known to be delirious, antipsychotics are no better than placebo. That begs the question, what's the harm in missing the diagnosis, in the absence of a treatment that works?

Girard: That is a very important question. At this point it's a question that I can only answer philosophically. There hasn't been a study that shows that if you identify delirium and then treat it that you will change long-term outcomes. But we also don't know that you can't change long-term outcomes. So I would say that, by assessing for and recognizing delirium, you may change your approach to that patient's management in ways that will benefit them.

For example, evaluating for and diagnosing delirium may make you more likely to use only light sedation, or even no sedation or medications that are less likely to contribute to delirium. Will it change their long-term cognitive outcomes? We don't know yet, because it hasn't been studied. Right now the only definitive risk factor we've identified for long-term

\footnotetext{
* Sean R Muldoon MD MPH, Kindred Healthcare, Hospital Division, Louisville, Kentucky.
} 
cognitive impairment after critical illness is duration of delirium in the ICU. Until we know any different, it seems appropriate to me-if not for any other reason, then at least for purely prognostic purposes-to assess for delirium.

If we're going to talk with families about the potential for long-term cognitive impairment in their critically ill loved ones, then what are we going to say about risk factors? The only thing that I know to say is that they've been delirious for a long time or they haven't been delirious for a long time. If a family member says to me, "I saw in the Wall Street Journal that patients end up with cognitive impairment months or years after being in the ICU. Is that going to happen to my husband?" then there's at least something that I can look to as a prognostic factor. How good am I at predicting that? I'm not that good, and I'd tell them that, but it's something I can talk with them about, prognostically.

Carson: From my own experience in delirium studies, the biggest help of the delirium screen is that it makes up for the fact that we can't recognize hypoactive delirium just by looking at a patient. The patient may have been agitated, received a drug, and now they look just fine. Their eyes are open and they're looking at you and even tracking you a little bit, so from the door or even the bedside they look fine. But if you do the delirium assessment, they're not there. A 2-minute assessment once a day by the nurse is enough to let you know that, whatever is going on with this patient medically, there is delirium present. And is there something we can do differently to make it not present? We may not be able to give a drug to make it go away, but we could certainly withhold drugs that may be contributing, or change the environment, or what have you.

Girard: That goes back to the comment about parachutes. ${ }^{1}$ There are cer- tainly some circumstances where assessing for delirium has benefits that are obvious but may never be tested or proven. Take, for example, a patient who is not delirious and then becomes delirious during their ICU stay; new-onset delirium can be a warning sign that prompts diagnostic assessments, which can benefit the patient.

Among older patients with sepsis, altered mental status or delirium is a very common presenting sign, and fever is less common than among younger patients. So if you're evaluating patients in or outside the ICU and they newly develop deliriumsomething you're often only going to detect if you're using a structured assessment tool-that tells you to look for a reason. Do they have an infection you haven't diagnosed yet? Do they have new renal failure or electrolyte abnormalities?

These potential changes in management are things that we're never going to study in a randomized controlled trial. Patients who have a urinary tract infection detected because they were noted to be delirious will not be randomized to treatment versus no treatment. That's the parachute idea: we don't need a randomized controlled trial to know that it's beneficial.

1. Smith GC, Pell JP. Parachute use to prevent death and major trauma related to gravitational challenge: systematic review of randomised controlled trials. BMJ 2003; 327(7429):1459-1461.

Carson: A topic Tim didn't have time to address is psychiatric outcomes of critical illness, like post-traumatic stress disorder. One thing that bothers survivors most is their memories of the hallucinations. We have more work to do to tie all these things together. However, I think that if we work to detect and prevent delirium, then maybe we'll spare some patients those very frightening hallucinations that tend to follow them for months down the road.
Snyder: $\dagger$ The point Neil made about sleep was really on target. I can tell you from my military flight medicine experience that sleep deprivation has a really big impact even on a healthy population, and if you consider it in a non-healthy population, you would infer that its effects are very profound. You talked about 2 long-term acute care (LTAC) studies ${ }^{1,2}$ that were about the same as far as results, both finding delirium in about half the patients shortly after LTAC admission. It's a shame we don't know the patients' cognitive status prior to LTAC admission. I wonder if it was actually coma: they were very sedated prior to LTAC admission, and what you're seeing in the first couple days after admission is that, even though the sedation was stopped-and typically it is, because we're trying to mobilize them and do all those other things-the delirium is just the effects of the sedation. I would have loved to see those studies assess patients for delirium 7 or 14 days after LTAC admission. I think that's a possible research project.

1. Weinert CR. Epidemiology of psychiatric medication use in patients recovering from critical illness at a long-term acute-care facility. Chest 2001;119(2):547-553.

2. Jubran A, Lawm G, Kelly J, Duffner LA, Gungor G, Collins EG, et al. Depressive disorders during weaning from prolonged mechanical ventilation. Intensive Care Med 2010;36(5):828-835.

Girard: As both a clinician and a researcher, I think that the CCI population is fascinating when it comes to evaluating them for delirium, because these patients aren't being heavily sedated, in contrast to patients with acute critical illness. What you find when you diagnose delirium in a patient with $\mathrm{CCI}$ is presumably more brain dysfunction due to their underlying illness rather than due to the treatments given in the ICU. $\dagger$ Lisa Snyder MD MPH, Select Medical, Me-
chanicsburg, Pennsylvania. 